\title{
Energy Efficiency in the Food Industry: A Systematic Literature Review
}

\author{
Angela Morandini Pradella ${ }^{\text {* }}$ \\ https://orcid.org/0000-0002-4624-5885 \\ Eduardo de Freitas Rocha Loures ${ }^{1,2}$ \\ https://orcid.org/0000-0002-1963-6186 \\ Sergio E. Gouvea da Costa ${ }^{1,3}$ \\ https://orcid.org/0000-0002-9882-5857 \\ Edson Pinheiro de Lima ${ }^{1,3}$ \\ https://orcid.org/0000-0001-9331-1569
}

\begin{abstract}
${ }^{1}$ Pontifical Catholic University of Paraná (PUCPR), Industrial and Systems Engineering Graduate Program, Curitiba, Paraná, Brazil; ${ }^{2}$ Universidade Tecnológica Federal do Paraná (UTFPR), DAELT, Curitiba, Paraná, Brazil; ${ }^{3}$ Universidade Tecnológica Federal do Paraná (UTFPR), DAELT, Pato Branco, Paraná, Brazil.
\end{abstract}

Received: 2018.11.05; Accepted: 2019.07.26.

*Correspondence: angelapradella@gmail.com; Tel.: +55-49-99981-3722 (A.M.P)

\section{HIGHLIGHTS}

- Indicators relating to four groups were identified in food industry and manufacturing literature.

- Results suggest greater concern of the food industry with the technologies of Industry 4.0.

- A differentiated approach in the food industry with method multi-criteria AHP and PROMETHEE.

- Energy indicators are poorly understood by the food industry, and therefore have not been implemented.

\begin{abstract}
Governments and private companies have increased efforts to identify effective actions for improving energy efficiency in manufacturing processes. The objective of this work is to improve the decision-making process by increasing the quality of information related to energy indicators in the food industry. This research involves developing a systematic literature review (SLR) to identify energy efficiency indicators in the food industry, which serve as inputs for a sectoral evaluation based on multicriteria techniques. The SLR identified six criteria evaluated by food industry experts, which form the proposed basis for evaluating the performance of related sectors. These criteria are: benchmarks, key performance indicators, framework, monitoring, ISO 50001, and information communication technologies (ICTs) in sectoral evaluations. The criteria were evaluated by experts using the Analytic Hierarchy Process (AHP), which prioritizes the most important food industry issues using an evaluation scale. Weights were attributed to each issue and positioned according to the Preference Ranking Organization Method for Enrichment Evaluations (PROMETHEE)
\end{abstract}


to evaluate each sector by the identified criteria. The evaluated criteria are applicable to the three sectors surveyed, with emphasis on the beverage sector. Among the evaluated criteria, ICTs were highlighted by Industry 4.0 as a concern for the food sector.

Keywords: energy efficiency; manufacture; food industries; analytic hierarchy process (AHP); preference ranking organization method for enrichment evaluations (PROMETHEE).

\section{INTRODUCTION}

The effects of climate change have put pressure on businesses to address green practices as well as financial performance to stay competitive with global requirements. As a primary consumer of natural resources with extensive carbon emissions, the manufacturing industry drives the need for sustainable manufacturing processes [1,2]. Energy is an increasingly critical factor for reducing carbon emissions and implementing cleaner production. Enhanced energy performance and its management may provide companies with a competitive advantage and an important strategic asset, enabling them to react with flexibility in change and development scenarios [3-5], and to maximize the use of their energy sources and assets, thereby reducing the cost of energy as well as that of its consumption [6].

Energy efficiency is a key phrase in modern industry that has become an essential factor for competitiveness, sustainability, and environmental performance [7]. Energy efficiency is defined as the output ratio for a given production device or system production for a facility operating under standard conditions or the volume of energy consumed by this production device, system, or facility to deliver its output [8].

Another way of measuring energy efficiency is using indicators to show the relationships between energy consumption and products. Indicators must consider the influences of economic and technical aspects [9]. In [10], performance indicators serve as metrics to determine whether systems are operating as designed and help to define progress in a given direction. This enables better tracking and control of energy consumption, which is extremely important to increase energy efficiency in production.

Analysis of energy indicators can also link consumption-related factors such as: energy efficiency, environmental policies, changes in energy prices, changes in international trade of energy-intensive intermediary or final products, and structural impacts stemming from economic cycles to develop tools for improving energy efficiency [11].

As discussed in [12], food is a basic human need. The food industry, therefore, is of major importance. Given the food industry's high energy consumption, food processing industries must adapt measures and implement actions to promote efficient energy use. According to [13], another indicator for assessing energy performance and efficiency in food production facilities is benchmarking, a method that consists of comparing Specific Energy Consumption (SEC) among facilities with similar characteristics [13].

This article aims to analyze energy efficiency mastery through a systematic literature review (SLR) of the methods and related indicators used in the manufacturing and food industries for three sectors (beverages, meats, and grains). Indicators were ranked by the level of importance allocated to each, with the objective of understanding the extent to which indicators identified by literature have been practically adopted by industry. The following assessment method was used: Analytic Hierarchy Process (AHP) and Preference Ranking Organization Method for Enrichment Evaluations (PROMETHEE). 


\section{MATERIAL AND METHODS}

The systematic review protocol was based on the stages proposed by [14,15], as shown in Figure 1.

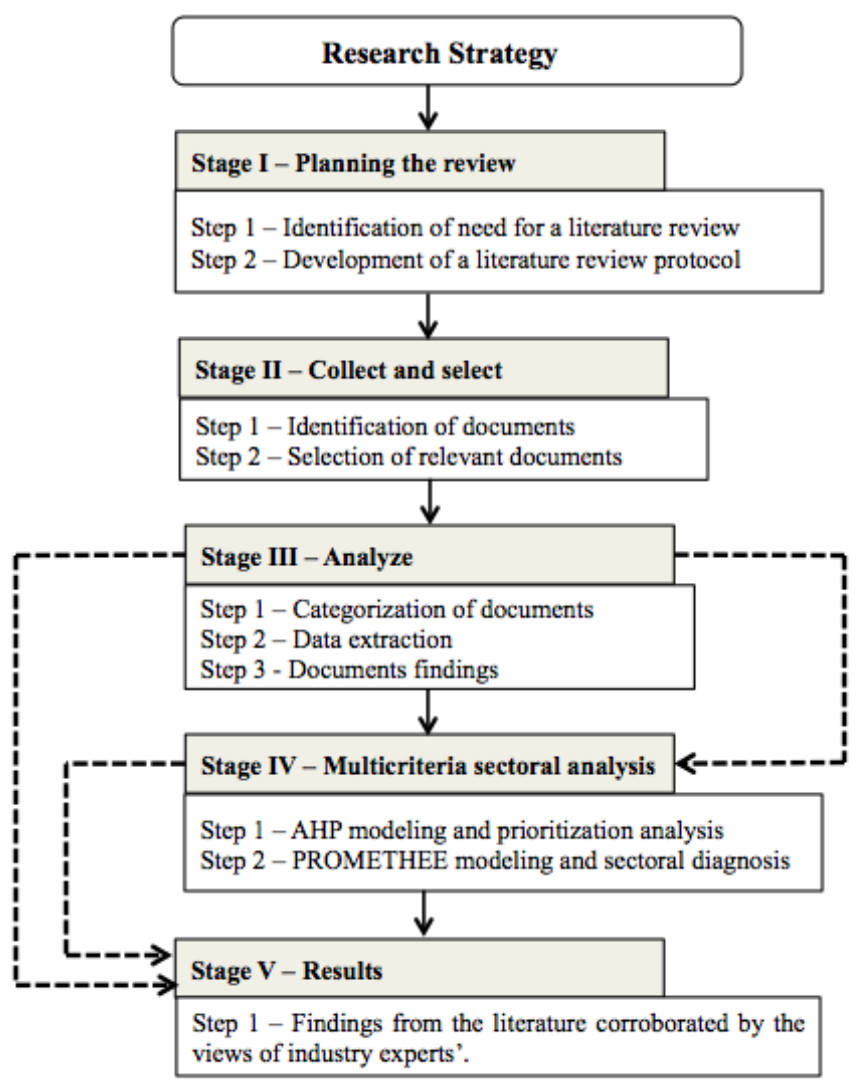

Figure 1: Research Strategy. Source: adapted from [14,15].

The first stage, called Planning the review, consists of two stages, Identification of need for a literature review and Development of a literature review protocol. The second stage, Collect and select, features two stages called Identification of documents and Selection of relevant documents. The third stage, Analyze, is comprised of Categorization of documents and Data extraction. The last stage, Results, which includes Document findings, involves reviewing all collected documents to extract pertinent information.

The literature review, described in Stage I, Step 1, Planning the review, is necessary because of the difficulty of tracking the advances and achievements related to energy efficiency initiatives that are currently adopted by the food industry.

Stage I, Step 2 addresses the development of the review protocol described in Figure 2, and details all stages associated with research document selection. As discussed in [14], the systematic review starts with identification of research keywords and terms, which were constructed based on study scope, literature, and discussions in the field. 


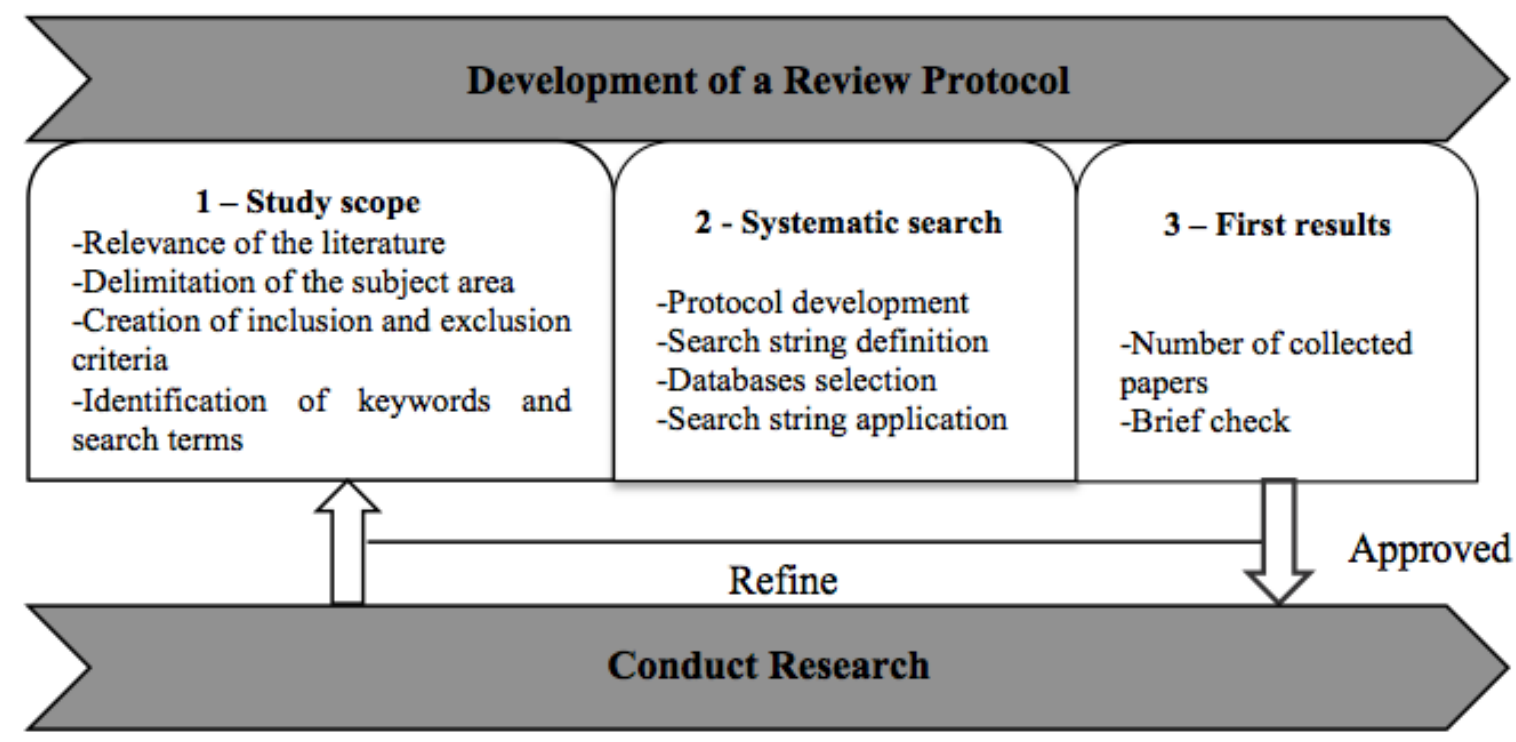

Figure 2: Development process of review protocol. Source: Adapted from [15].

The searches were based on the words energy management and indicator or (KPI) with "AND" and "OR" as Boolean operators between keywords and search fields. Strings defined for the search were: Title: ("energy management" AND "indicator" OR "KPI") OR Abstract: ("energy management" AND "indicator" OR "KPI") OR Keywords: ("energy management" AND "indicator" OR "KPI"). These boundaries did not guarantee documents focused on the research topic, which led to establishment of exclusion criteria to exclude papers unrelated to energy issues in the context of production or the food industry.

English was chosen as the language because of the high number of English language publications, which provided a higher number of relevant documents in the survey. A twenty-one year time window (1996 to present) was chosen. This timeframe is directly linked to an increase of publications on energy topics that began in 1996. Figure 3 provides a detailed view of the protocol through which search criteria and requirements were defined.

Three online databases were used to search for published articles: Scopus, Web of Science, and Science Direct. Database searches are conducted in similar formats, but must be customized for search engine particularities.

\section{Collect and select}

Execution of the search protocol, described in Figure 2, followed Stage II collection and selection, as per Figure 1. Documents were identified using a preliminary document survey to select relevant documents in each databases.

\section{Analyze}

Stage III analyze is the next step, outlined in Figure 1. Searching all three databases resulted in 448 documents, which were categorized as per Step 1. Documents were processed using an Excel spreadsheet to remove duplicate materials as well as those that were inaccessible in the database. This data extraction step (Step 2) yielded 328 papers. Next, titles and abstracts were read to select and discard documents meeting the exclusion criteria defined in the research protocol. A total of 49 articles dealing with energy management or efficiency in the manufacturing or food industries were thus selected for complete reading for execution of Step 3 document findings. Table 1 displays the protocol used for the database survey. 
Table 1: Review Protocol. Source: Author.

\begin{tabular}{cc}
\hline Item & Description \\
\hline $\begin{array}{c}\text { Keywords } \\
\text { Boolean } \\
\text { Operators }\end{array}$ & $\begin{array}{r}\text { "energy management" AND "indicator" OR "KPI" OR "energy } \\
\text { efficiency" AND "indicator" OR "KPI" }\end{array}$ \\
$\begin{array}{c}\text { Search fields } \\
\text { Exclusion }\end{array}$ & $\begin{array}{c}\text { title; abstract; keywords in first search string; title in second search } \\
\text { string } \\
\text { criteria }\end{array}$ \\
$\begin{array}{c}\text { Language } \\
\text { Publication } \\
\text { type }\end{array}$ & Articles not dealing with energy issues in the context of production \\
Period & English \\
\hline
\end{tabular}

Table 2 shows the strings adopted within each of the database to return the most effective papers for this research.

Table 2: Modification of the search string by database. Source: Author.

\begin{tabular}{cl}
\hline \multicolumn{1}{c}{ Database } & \multicolumn{1}{c}{ Search String } \\
\hline \multirow{3}{*}{ Science Direct } & TITLE ("energy management" AND "indicator" OR "KPI") OR \\
& -KEYWORDS ("energy management" AND "indicator" OR "KPI") \\
& TITLE ("energy efficiency" AND "indicator" OR "KPI") \\
& TS=((("energy management") AND ("indicator") OR ("KPI")))) AND \\
Web of Science & Language: (English) AND Types of documents: (Article) \\
& TI=((("energy efficiency") AND ("indicator") OR "KPI")))) AND \\
& Language: (English) AND Types of documents: (Article) \\
& TITLE ("energy management" AND "indicator" OR "KPI") OR ABS \\
& ("energy management" AND "indicator" OR "KPI") OR KEY \\
& ("energy management" AND "indicator" OR "KPI" AND (LIMIT-TO) \\
& (LANGUAGE, "English")) AND (LIMIT-TO (SRCTYPE, "j") TITLE \\
& "energy efficiency" AND "indicator" OR "KPI") AND DOCTYPE (ar) \\
& AND PUBYEAR>1995 \\
\hline
\end{tabular}

In the Analyze phase, the selected articles were categorized by publication (Table 3). The applied filters identified the most relevant papers for developing better understanding of energy efficiency indicators across the manufacturing and food industries. 
Table 3: Journals with the highest numbers of publications used in this survey. Source: Author.

\begin{tabular}{cc}
\hline Publication & $\mathbf{N}^{\circ}$ of Papers \\
\hline Energy & 50 \\
Journal of Cleaner Production & 41 \\
Energy Policy & 27 \\
Applied Energy & 25 \\
Energy Conversion and Management & 23 \\
Energy and Buildings & 19 \\
Energy Procedia & 14 \\
Renewable and Sustainable Energy & \\
Reviews & 11 \\
Renewable Energy & 10 \\
Energy Efficiency & 7 \\
Energy Economics & 5 \\
\hline
\end{tabular}

\section{Multicriteria sectoral analysis}

The AHP method was applied in Stage IV, Step 1 for multicriteria sectoral analysis, which involved expert assessment as well as modelling and analysis. Food industry assessment criteria was prioritized and weighted for input to the second method-PROMETHEE. In Step 2, PROMETHEE was used for diagnostic modelling and assessment of each sector according to the six criteria.

\section{Results}

Stage 5 results involved findings from the literature as well as specialist inputs from each of the three food industry sectors. Standouts from the literature indicated a criteria that were both assessed in literature and practically applied by specialists in industry.

\section{RESULTS AND DISCUSSION}

\section{Sector Analysis}

The literature review informed mapping of energy efficiency indicators used in both general manufacturing and in the food industry and identified gaps between the sectors. From the results of the literature analysis, six criteria were identified for the research design of this study, which were evaluated by specialists from each sector.

Different aspects of energy efficiency integration in production management (measurement, control, improvement, and enablers) were analyzed using literature focused on general industry and the food industry specifically. Politicians and companies have discussed energy efficiency for decades; however, the results highlighted in this section indicate numerous differences among sectors in terms of advances and adoption of indicators.

As per [6], Table 4 summarizes elements identified in literature related to manufacturing. Elements relating to the food industry included measurement, control, improvements, and facilitators for production processes. 
Manufacturing-related KPIs are widely available as a form of measurement, but these do not apply to plant-specific food industry processes because of the inherent complexity of monitoring variables. KPIs are linked to energy content and productivity; productivity is represented by $P / E$, where $P$ represents the quantity produced and $E$ represents the quantity of energy in $\mathrm{kW}$. In this sense, the literature indicates a major gap; KPIs are rarely used in the food industry. Using KPIs may significantly improve energy efficiency in plants and reduce the energy consumption of the production process.

Benchmarking is widely adopted in manufacturing to track energy consumption and is used for comparisons among sectors or countries. Benchmarking in the food industry takes the form of overall plant assessments, but its adoption is of low significance in the food sector. The lack of benchmarking in the food sector applies to both plants and production equipment; therefore, it is difficult to compare plants producing the same types of products.

Table 4: Approaches in manufacturing and food industry and the gaps identified in literature. Source: adapted from [6].

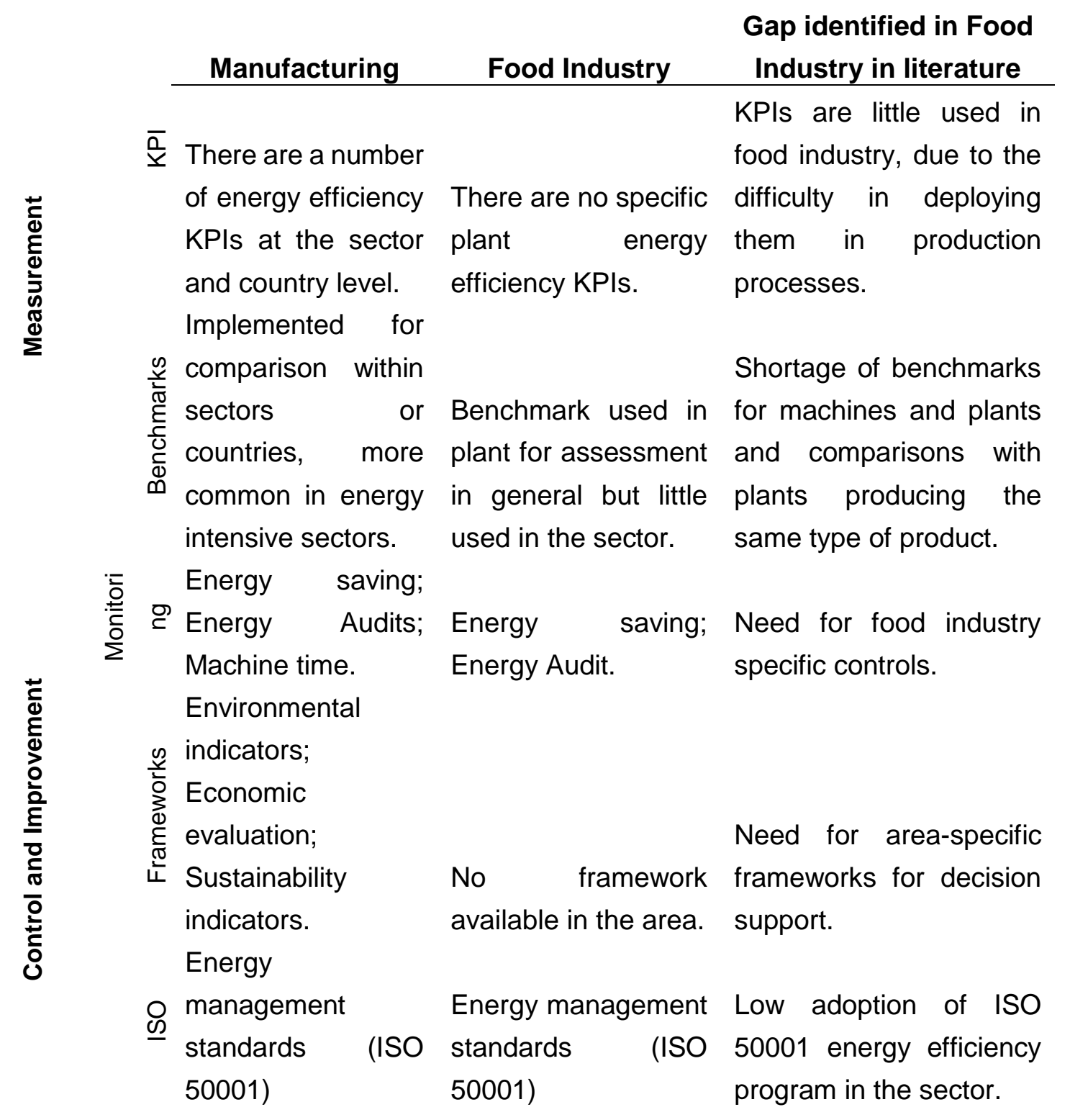


Angela, M.P.; et al.

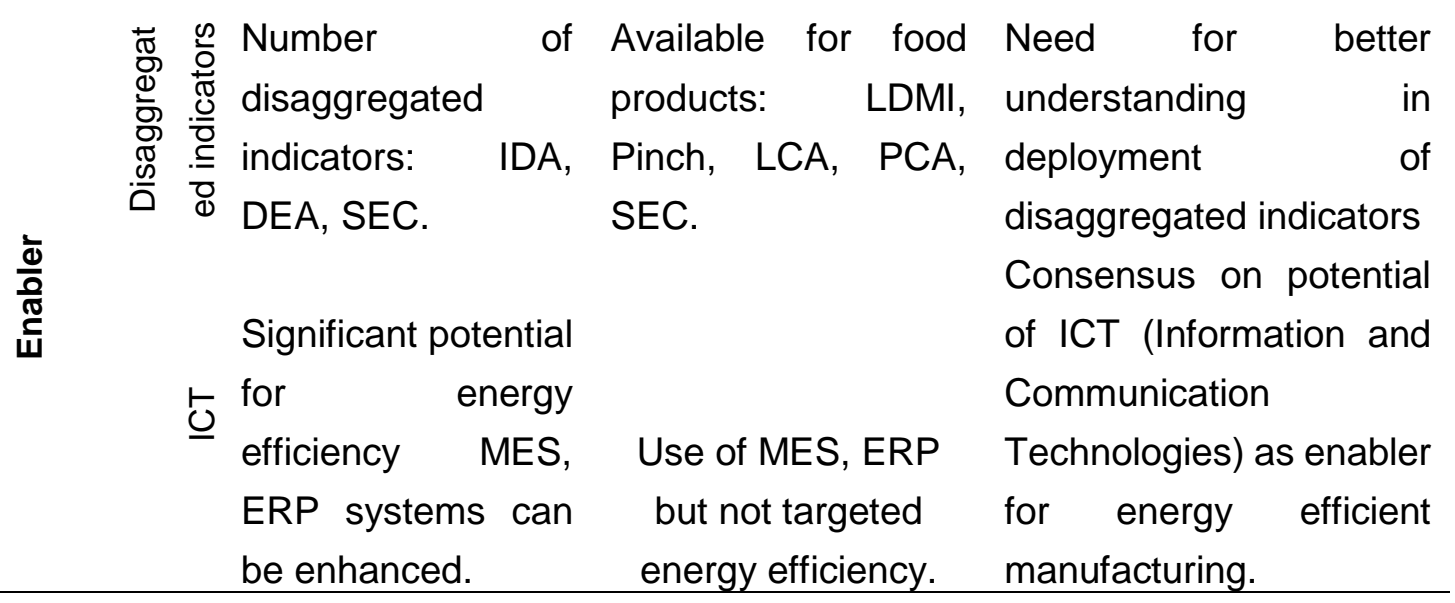

There are many methods and tools for monitoring and control, such as energy audits and energy savings in both the manufacturing and food industries. However, these studies have no controls (tools, techniques, indicators, and metrics) that can be replicated. These were only adopted for case studies, to assess specific processes. Thus, the results are not relevant for other plants in the food sector. To improve energy efficiency, the food industry needs conceptual structures and tools for assessment, support, and decision-making.

There are several decision-making support systems (e.g., multi-criteria decision support) that can be adopted and enhanced to optimize efficiency. Additionally, there are references available related to assessment of energy efficiency metrics (e.g., indicators in sustainability and economic assessment). For companies benefiting from decision-making support tools, plant level production management must be adapted to address energy efficiency issues. Plant assessment frameworks are not available for the food industry to guide the cost-benefit assessment of investing in the energy efficiency and decision-making support tools that have been confirmed by the literature and by general industry.

The energy management ISO is available for manufacturing and food production plants, but most production planning and control systems do not integrate energy efficiency and relevant performance standards. The broadest assessments of these standards meet the needs of energy-intensive manufacturing facilities by addressing production energy management (including aspects like adaptation, benefits, and cost of standards). Food industry facilities that are not energy-intensive, however, do not benefit from adopting ISO standards for energy efficiency. The ISO standards represent an important enabling factor, as comparability and competitiveness in production energy management can be improved.

Information and Communication Technologies (ICT) can help manage and reduce energy consumption in manufacturing processes. They may help to control production processes and assess potential energy-saving investments. Company ICT infrastructure may consist of different systems such as the Enterprise Resource Planning Systems (ERP), Manufacturing Execution Systems (MES), or Supply Chain Management Systems (SCM). The IC proposal is addressed in Industry 4.0 and the food industry will need to develop and improve its processes through an inter-equipment communication channel.

The gaps identified in the literature (Fig. 6) concerning the food industry were corroborated by specialist evaluations for each sector. Degrees of importance were assigned to the six criteria in the AHP method and each sector was ranked by the criteria 
using the PROMETHEE method.

Notably, the perception of Brazilian specialists is different from that expressed in the literature because of different nomenclature and indicators that apply only to Brazilian food production processes. These differences made the task of comparing measurable indicators more complex because of the different terminology used by Brazilian companies. Despite this limitation, the assessment was conducted using the indicators with common definitions in literature and industry: benchmarking, KPI, framework, monitoring, ISO 50001, and ICT.

For the assessment, specialists were selected from the beverage, meat, and grain sectors, which are representative of the Brazilian food production industry. The next section uses the AHP method to evaluate prioritization vectors attributed to each of the identified criteria.

\section{Sector Analysis using the Analytic Hierarchy Process (AHP)}

The AHP method is used to generate priorities by comparing discrete and continuous pairs [16]. The method is based on three principles of analytical thinking: (a) construction of hierarchies, (b) establishment of priorities, and (c) logical consistency testing [17]. For the food industry, the problem was structured hierarchically by objective, criteria, and sub-criteria. To define the importance of two elements at the same hierarchical level, comparative matrixes are built and preference scores are attributed to each element according to a scale developed by [18].

Values were assigned in AHP using the Super Decisions software tool, a tool that provides a user-friendly interface for assessing inputs and compatibility with PROMETHEE, which was applied in the second part of sector evaluation. Figure 3 shows the AHP model structure adopted by the proposed evaluation. First, six sub-criteria were identified: BC (Benchmarking), KPI (Key Performance Indicator), FR (Framework), MON (Monitoring), ISO 50001, and ICT (Information and Communication Technologies). Next, weights for each sub-criterion were entered into PROMETHEE.

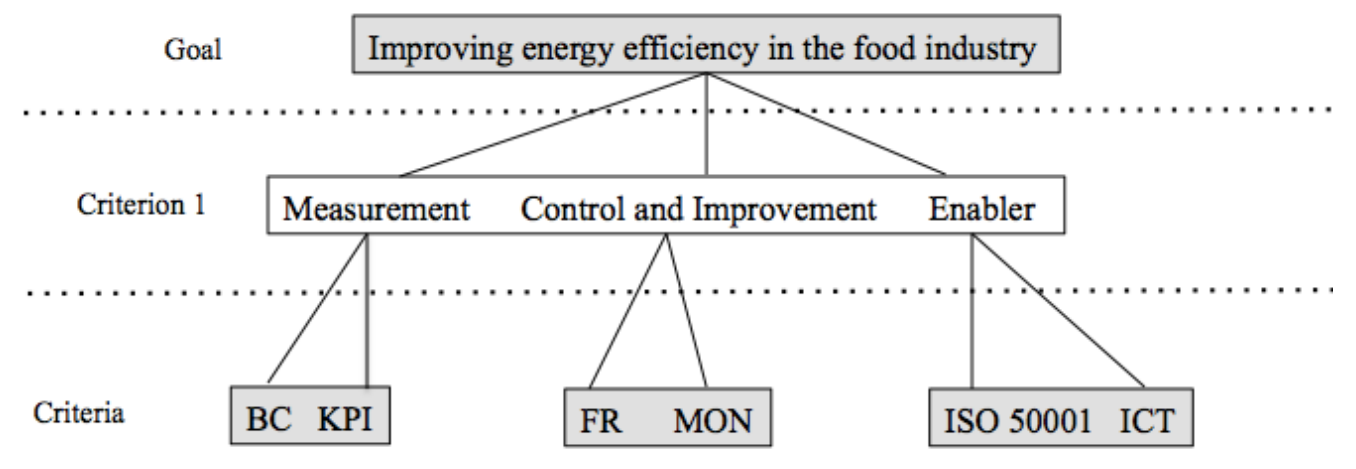

Figure 3: AHP structure. Source: Author.

The results from AHP are graphically depicted in a radial format (Chart 1). As the chart shows, ICTs are the most important indicator in the food industry. ICTs are used to qualify industry competencies and concerns in Industry 4.0, resulting in a high prioritization factor for ICTs in the food industry. 


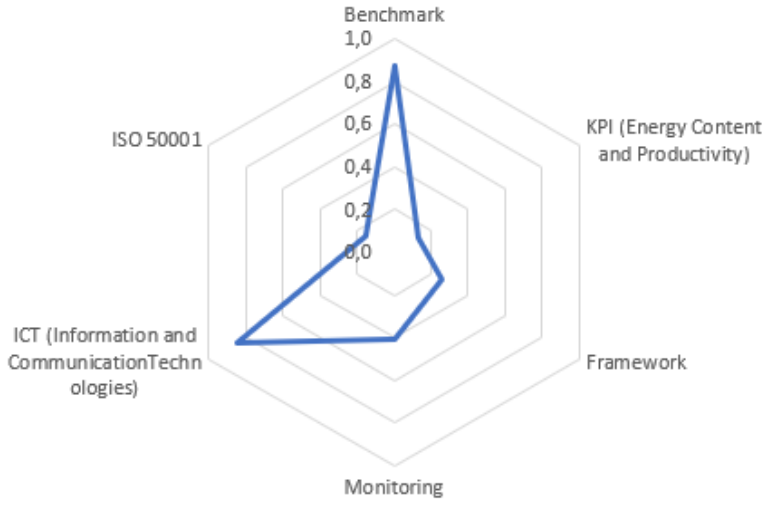

Chart 1: Analysis of results by AHP. Source: Author.

The results indicate that benchmarking assessment is also a high prioritization vector in the food industry. Benchmarking can be used to compare machinery, plants, or sector efficiencies. Monitoring was also identified as an industry concern with a medium prioritization vector related to tracking production processes and detecting failure occurrences.

For KPIs and ISO, the resulting vectors show lower concerns, confirming the assessors' perceptions. The framework shows a weight near three, which likely indicates a lack of knowledge of its deployment in the industry. After performing diagnostics with AHP, weight information was used for assessment by the PROMETHEE method, as shown in Figure 4.

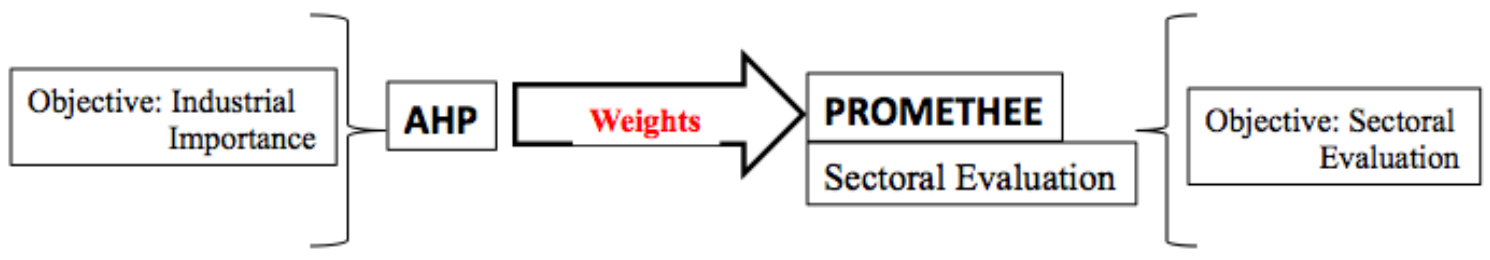

Figure 4: Objective for applying two methods: AHP and PROMETHEE. Source: Author.

\section{Sector Assessment using the PROMETHEE method}

PROMETHEE is based on two phases: building over-classification relationships, which add information among alternatives and criteria, and exploring these relationships to support decision-making [19]. The weights adopted from the AHP method (prioritization vectors) were transferred to the PROMETHEE method based on the fact that the higher the weight, the more important the criteria.

PROMETHEE's preference structure is based on pairwise comparisons between two alternatives of a given criteria. The six evaluated criteria evaluated are highlighted in Item 1 and were assessed on a weight scale of 1 to 9 points in AHP. Item 2 has an ascending scale with fields $(\min / \max )$. Field "weight" contains weights from AHP. Item 3 provides statistics based on parameter definitions and will be used to describe the method's results. The final Item 4 indicates the three evaluated sectors (beverages, meats and grains) and positions the results of each sector with respect to the evaluated criteria. Figure 5 shows the results obtained with PROMETHEE. 


\begin{tabular}{|c|c|c|c|c|c|c|c|c|}
\hline & \multicolumn{2}{|l|}{ Scenario1 } & Benchmark & KPI & Framework & Monitoring & ISO 50001 & ICT (Informa... \\
\hline & \multicolumn{2}{|l|}{ Unit } & 9-point & 9-point & 9-point & 9-point & 9-point & 9-point \\
\hline & \multicolumn{2}{|l|}{ Cluster/Group } & & & & & & \\
\hline & \multicolumn{2}{|l|}{ Preferences } & & & & & & \\
\hline & \multicolumn{2}{|l|}{ Min/Max } & $\max$ & $\max$ & $\max$ & $\max$ & $\max$ & $\max$ \\
\hline & \multicolumn{2}{|l|}{ Weight } & 8,70 & 1,27 & 2,66 & 4,18 & 1,47 & 8,48 \\
\hline & \multicolumn{2}{|l|}{ Preference Fn. } & Level & Level & Level & Level & Level & Level \\
\hline & \multicolumn{2}{|l|}{ Thresholds } & absolute & absolute & absolute & absolute & absolute & absolute \\
\hline & \multicolumn{2}{|l|}{ - Q: Indifference } & 0,00 & 1,00 & 1,00 & 1,00 & 1,00 & 1,00 \\
\hline & \multicolumn{2}{|l|}{ - P: Preference } & 2,00 & 2,00 & 2,00 & 2,00 & 2,00 & 2,00 \\
\hline & \multicolumn{2}{|l|}{-S: Gaussian } & $\mathrm{n} / \mathrm{a}$ & $\mathrm{n} / \mathrm{a}$ & $\mathrm{n} / \mathrm{a}$ & $\mathrm{n} / \mathrm{a}$ & $\mathrm{n} / \mathrm{a}$ & $\mathrm{n} / \mathrm{a}$ \\
\hline & \multicolumn{2}{|l|}{ Statistics } & & & & & & \\
\hline & \multicolumn{2}{|l|}{ Minimum } & 8,00 & 1,00 & 1,00 & 1,00 & 2,00 & 8,00 \\
\hline & \multicolumn{2}{|l|}{ Maximum } & 9,00 & 2,00 & 9,00 & 9,00 & 2,00 & 9,00 \\
\hline & \multicolumn{2}{|l|}{ Average } & 8,33 & 1,33 & 4,00 & 6,00 & 2,00 & 8,33 \\
\hline & \multicolumn{2}{|l|}{ Standard Dev. } & 0,47 & 0,47 & 3,56 & 3,56 & 0,00 & 0,47 \\
\hline & \multicolumn{2}{|l|}{ Evaluations } & & & & & & \\
\hline$\square$ & Beverage Sector & $\square$ & extreme & equal & weakly & very strongly & weakdy & very strongly \\
\hline$\square$ & Meat Sector & $\square$ & very strongly & equal & extreme & equal & weakly & extreme \\
\hline$\square$ & Grain Sector & $\square$ & very strongly & weakly & equal & extreme & weakly & very strongly \\
\hline
\end{tabular}

Figure 5: PROMETHEE Results. Source: Author.

Figure 6 shows the ranking of the three assessed sectors: beverages, meat, and grain, by the six criteria. This assessment positioned each sector by the criteria using the weights attributed to each. Generally, the beverages sector was the most compliant with the assessed criteria, resulting in a positive flow. The other two sectors displayed negative flows, but with similar values, indicating that the importance ascribed to the criteria was low.

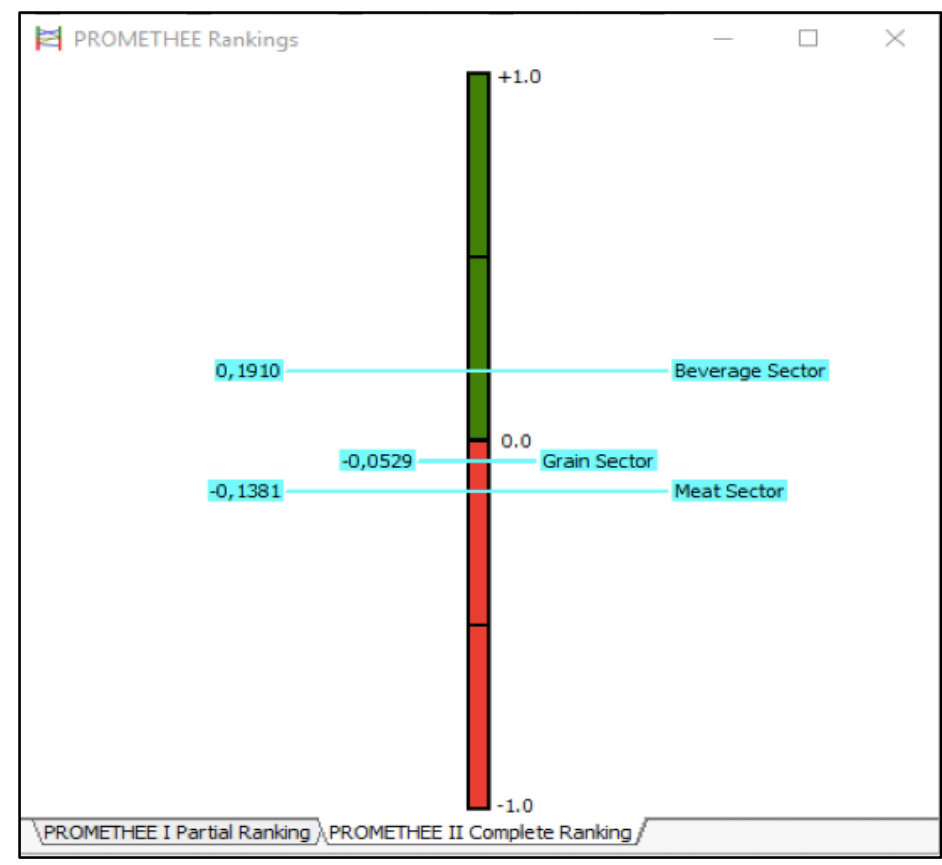

Figure 6: PROMETHEE Rankings. Source: Author.

For each alternative, two indices are calculated from the preference indices: the positive flow $(\Phi+)$ represents the extent to which a given alternative is better than the others, while the negative flow $(\Phi-)$, expresses the extent to which the alternative is exceeded by others, 
with the resulting "Phi" for each criteria. Figure 7 shows that the beverages sector has a Phi + , corroborating the AHP results that indicate this sector has the highest scores for the assessed criteria.

\begin{tabular}{|c|c|c|c|c|c|}
\hline \multicolumn{4}{|c|}{ PROMETHEE Flow Table } & \multicolumn{2}{|c|}{$\square$} \\
\hline Rank & actio & & Phi & Phit+ & Phi- \\
\hline 1 & Beverage Sector & $\square$ & 0,1910 & 0,2407 & 0,0496 \\
\hline 2 & Grain Sector & $\square$ & $-0,0529$ & 0,0781 & 0,1309 \\
\hline 3 & Meat Sector & $\square$ & $-0,1381$ & 0,0993 & 0,2374 \\
\hline
\end{tabular}

Figure 7: PROMETHEE Flow Table. Source: Author.

The PROMETHEE method enables sensitivity analysis to be performed by simulating different scenarios. Figure 8 shows the results of this simulation. Increased use of ICT can be projected in the coming years due to Industry 4.0 adoption by companies, suggesting that the beverages sector, followed by the meat sector, will be better supported by these technologies. This would enhance understanding and adoption of the other indicators evaluated in this study.

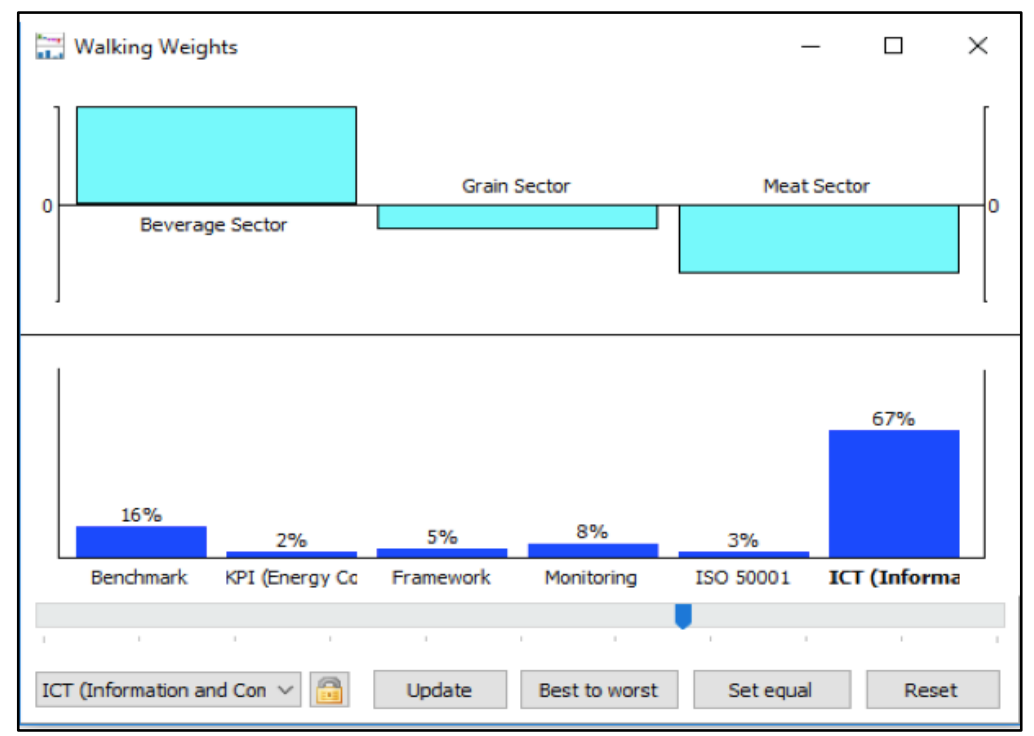

Figure 8: Visual PROMETHEE-ICT scenario. Source: Author.

Another simulated scenario evaluates the impact of monitoring across the three sectors. The increase led to slight growth in the beverages sector and marked growth for the grains sector, while the meat industry decreased due to multiple controls based on the high volume of product export, as shown in Figure 9. 


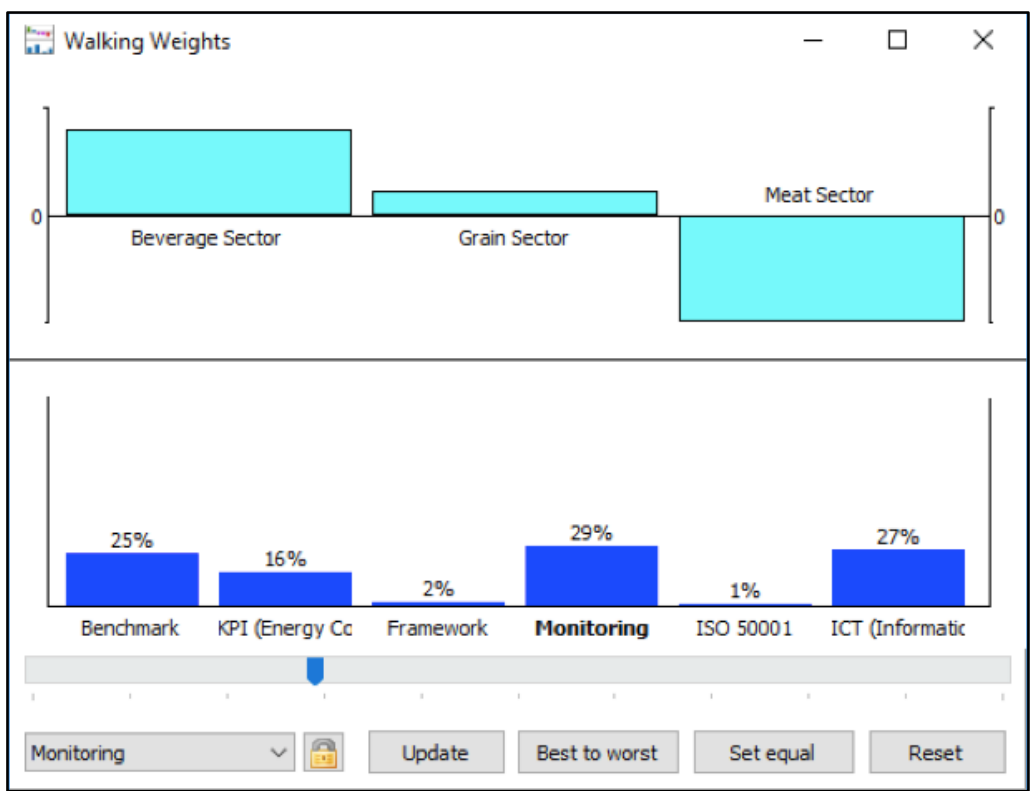

Figure 9: Visual PROMETHEE—Monitoring scenario. Source: Author.

The results obtained with both methods, AHP and PROMETHEE, allowed each of the six criteria to be organized by weight to define indicators for the three food industry sectors, according to the literature.

\section{CONCLUSION}

The objective of this paper was to present a systematic literature review for the field of energy efficiency measurement and indicators in the food industry, as well as sector assessment by specialists from the beverages, meats and grains sectors. The main contribution of this survey is identifying energy indicators used in the food industry as well as the six criteria assessed in each sector. These criteria were considered by the literature as the most important for understanding industry evolution of energy efficiency. The assessment was performed using the AHP and PROMETHEE methods, which facilitated results ranking for each sector. The assessments emphasized that the topic of energy efficiency is still unclear for companies and that the understanding of the indicators that may be adopted for the measurements remains low.

There were a few notable characteristics identified by this survey. Although in both manufacturing and the food industry, process measurement, control, and enhancement in solutions are available, advances in these improvements are far from the implementation stage, because of the factors highlighted in the discussion section. In many cases, solutions are inadequate for energy management in production at the company, plant, and process levels. There is a major gap between available solutions available and effective implementation by companies.

To reduce the gap between theory and practice, research should focus on improving the perception and understanding of the indicators identified in the literature and their applications in the food industry. It is necessary to determine when, where, and how plant level energy efficiency KPIs should be measured and displayed. The use of standardized KPIs may help the food industry to better implement and adopt benchmarking. 
Assessing each of the three sectors with the AHP and PROMETHEE methods reveals each sector's performance for each of the identified criteria. The results identified a gap in understanding and applicability of available indicators, with a low level of concern for the importance of Industry 4.0, which may promote energy and process efficiency.

The work is constrained by the number of food industry sectors evaluated; interviews could be increased to yield results with more applicability. Proposing energy management systems that utilize KPIs helps to increase future research opportunities that can be replicated across different sectors of the food industry. Future research will improve the understanding of indicators, their applicability, and the level of importance attributed to energy efficiency in the food sector. Because the field is broad, there is a range of opportunities to develop unique tools such as ICTs or frameworks. This article contributes by surveying indicators to be used by the food industry as well as by providing sector assessment by specialists. The results achieved through the evaluations suggest that adopting process indicators is a key concern.

Acknowledgments: The present work was carried out with the support of the Coordination of Improvement of Higher Education Personnel-Brazil (CAPES)-Financing Code 001.

\section{REFERENCES}

1. Ntabe, E.N.; Lebel, L.; Munson, A.D.; Santa-Eulalia, L., 2015. A. A systematic literature review of the supply chain operations reference (SCOR) model application with special attention to environmental issues. Int. J. Prod. Econ., 2015, 169, 310-32.

2. Ngai, E.W.T.; Chau, D.C.K.; Poon, J.K.L.; To, C.K.M. 2013. Energy and utility management maturity model for sustainable manufacturing process. Int. J. Prod. Econ., 2013, 146, 453-64.

3. Laitner, J.A. 2013. An overview of the energy efficiency potential. Environ. Innov. Soc. Transit., 2013, 9, 38-42.

4. Dobes, V. New tool for promotion of energy management and cleaner production on no cure, no pay basis. J. Clean. Prod., 2013, 39, 255-64.

5. Pye, M.; McKane, A. Making a stronger case for industrial energy efficiency by quantifying non-energy benefits. Resour. Conserv. Recycl., 2000, 28, 171-83.

6. Bunse, K.; Vodicka, M.; Schoensleben, P.; Brülhart, M.; Ernst, F.O. 2011. Integrating energy efficiency performance in production management e gap analysis between industrial needs and scientific literature. J. Clean. Prod., 2011, 19, 667-79.

7. Semkov, K.; Mooney, E.; Connolly.; M, Adley, C. Efficiency improvement through waste heat reduction. Appl. Therm. Eng., 2014, 70, 716-22.

8. Wojdalski, J.; Grochowicz, J.; Drózdz, B.; Bartoszewska, K.; Zdanowska, P.; Kupczyk, A.; Ekielski, A.; Florczak, I.; Hasny, A.; Wójcik, G. Energy efficiency of a confectionery plant - Case study. J. Food Eng., 2015, 146, 182-91.

9. Martínez, C.I.P. Analysis of energy efficiency development in the German and Colombian food industries. Int. J. Energy Sect. Manag., 2010, 4(1), 113-36.

10. May, G.; Taisch, M.; Prabhu, V.; Barletta, I. Energy related key performance indicators - state of the art, gaps and industrial needs. Advances in production management systems. Sustainable production and service supply chains. Ad. Inf. Communication Technology, 2013, 414(1), 25767.

11. Eichhammer, W.; Mannsbart, W. Industrial Energy Efficiency - Indicators for a European crosscountry comparison of energy efficiency in the manufacturing industry. Energy Policy, 1997, 25(7-9), 759-72.

12. Lin, B.; Xie, X. Factor substitution and rebound effect in China's food industry. Energy Convers. Manag., 2015, 05, 20-9. 
13. Reindl, D.T.; Jekel, T.B.; Elleson, J.S. In Book Industrial refrigeration energy efficiency guidebook. Madison: The University Wisconsin IRC, Industrial Refrigeration Consortium, 2005.

14. Tranfield, D.; Denyer, D.; Smart, P. The modern approach to industrial maintenance management. Br J. Manag., 2003, 14, 207-22.

15. Ruschel, E.; Santos, E.A.P.; Loures, E de F.R. Industrial maintenance decision-making: A systematic literature review. J. Manuf. Syst., 2017, 45, 180-94.

16. Saaty, R.W. The analytic hierarchy process e what it is and how it is used. Math. Model, 1987, 9, 161-76.

17. Huyen Do, T.T.; Schnitzer, H.; Le, T.H. A decision support framework considering sustainability for the selection of thermal food processes. J. Clean. Prod., 2014, 78, 112-20.

18. Saaty, T.L. Decision making with the analytic hierarchy process. Int J. Serv. Sci., 2008, 1(1), 8398.

19. Almeida, A. T. de. Processo de Decisão nas Organizações, 1nd ed.; Publisher: Atlas, São Paulo, Brazil, 2013; Volume 1. BY NC) license (https://creativecommons.org/licenses/by-nc/4.0/). 